\title{
The Evolution of Vector Magnetic Fields and the Origin of Coronal Mass Ejections (CMEs)
}

\author{
Jun Zhang, Guiping Zhou and Jingxiu Wang \\ ${ }^{1}$ National Astronomical Observatories Chinese Academy of Sciences, Beijing 100012, China \\ email:wjx@ourstar.bao.ac.cn; zhougp@ourstar.bao.ac.cn; zjun@ourstar.bao.ac.cn
}

\begin{abstract}
Coronal mass ejections (CMEs) are intrinsically associated with magnetic structures and evolutions in the solar photosphere. Based on the analysis of vector magnetic field data, we found that: 1, magnetic flux cancellation is the most universal magnetic change in the course of CME onset; 2, new flux emergence also plays an important role in CME origination; 3, interaction and reconnection of flux systems with opposite sign helicity is another key element in the magnetism of CME initiation.
\end{abstract}

Keywords. Sun: magnetic fields, Sun: coronal mass ejections (CMEs)

\section{Introduction}

Coronal mass ejections (CMEs) are known as the most spectacular form of solar activity. An equivalent phenomenon has just begun to be identified in the other astrophysical objects. Despite of the significant improvement of coronagraphic and non-coronagraphic (see Hudson \& Cliver 2001) observations of CMEs in recent years, the physics of their initiations remain unsettled. The low coronal observations (in X-rays and EUV) are very easily associated with a limb CME, but missing good measurements of surface magnetic field. Magnetic flux erupting in CMEs are believed to connect (at least initially) to the photosphere without question. Therefore CMEs' initiations can not be understood properly without reference to the surface magnetism (Feynman \& Martin 1995).

CMEs always are associated with filaments' eruptions. A filament will erupt when new magnetic flux emerges within or adjacent to the unipolar magnetic fields astride the filament in an orientation favorable for reconnection. Flux cancellation in the vicinity of a neutral line is suggested to be a necessary condition for a filament's formation and eventually for its eruption. The association of flares and canceling magnetic fields was first noted by Martin, Livi , \& Wang (1985) and was discussed later by Livi et al. (1989) and(Wang, \& Shi 1993).

Many CMEs contain helical magnetic structures (Dere et al. 1999), which has been proved by in situ observations near 1 AU in magnetic clouds (Burlaga \& Behannon 1982). CMEs are thought to originate from an over-accumulation of magnetic helicity and to take off the magnetic helicity from the Sun into the interplanetary space (Rust \& Kumar 1994; Low 1996; van Driel-Gesztelyi et al. 1999). Thus, to understand the buildup processes of magnetic helicity in CMEs becomes a central issue in CMEs' studies.

Magnetic helicity quantifies the topology complexity which constrains the minimum energy status of a given flux system during its evolution. Thus, it has been taken as one of the necessary ingredients in flare/CME models. Many other elements, such as the magnetic connectivity, magnetic non-potentiality (vertical currents, magnetic shear), magnetic flux evolution (flux emergence and cancellation), and dynamics (coherent pore 
and sunspot motion, sunspot rotation), describe other, more or less, independent ingredients of the flare/CME magnetism (Wang, 2002).

\section{Magnetic flux cancellation and CME.}

Magnetic flux cancellation is described as the mutual flux disappearance in closelyspaced magnetic fields of opposite polarities. The opposite polarity flux involved in the flux cancellation is referred to as a Canceling Magnetic Feature. Reconnection in the lower atmosphere was implied.

On July 14, 2000, a great solar flare with X-ray importance of X5.7 launched near the disk center in active region, NOAA 9077 . The flare was accompanied by a giant filament eruption and an extended Earth-directed CME. The arrival at Earth of the massive electrified gas cloud from this CME caused vivid aurora on July 16. Figure 1 shows the general appearance of the active region, NOAA 9077, and the various manifestations of the event. The arrow at Panel 'A' indicates a filament. Seen from the TRACE $1600 \AA$ movie, we found that the brightening first appeared from the region shown by a window at Panel 'B', then the bright material (the arrow at Panel 'B') moved along the channel (shown by a dot curve) of the filament to the right. Several hours before the filament eruption, some bright points (or patches) had already appeared on both sides of the filament. The arrow at Panel ' $\mathrm{C}$ ' indicates one main bright patch. It enlarged along the filament to form a bright ribbon. This bright patch was also identified in TRACE 195 $\AA$ and $1600 \AA$ images (see Panels 'A' and 'B'). Comparing the magnetogram with the $\mathrm{H} \alpha$ filtergram in the figure, we noticed that the bright patch was located at the region (indicated by the arrow at Panel ' $D$ ') where a pair of opposite polarity fields was closely contacting and canceling. The eruption of the filament and the onset of the flare were accompanied by an extended halo CME, see the running difference image of LASCO C2 in Panel ' $\mathrm{F}$ '.

To illustrate the process of the filament eruption and the flare onset, we present in Figure 2 the time sequence of TRACE $195 \AA$ images. The filament (indicated by the arrow at 09:30:15 UT) was apparently consisted of two twisting threads (indicated by the upper two arrows at 09:46:06 UT), one was thicker and diffusive, and the other, thinner and compact. At an inflection point of the filament, the filament appeared to be bifurcated (see the lower arrow at 09:46:06 UT). At first, the thinner one of the two threads was cut off at the point, indicated by an arrow in the frame of 09:48:17 UT, then the two threads broke off. At the broken point, a bright flare patch (shown by the arrow at 10:09:19 UT) appeared while the filament began to erupt. The filament was torn into two pieces from the broken point. The upper piece seemed to be stable, while the lower one rose from one end closed to the broken point with another end fixed in place. Several minutes later, another flare patch (see the arrow at 10:11:39 UT) appeared near the fixed end of the filament. This flare patch became larger and larger, meanwhile the whole body of the lower piece of filament started to rise and erupt. The erupted piece appeared rotating, and two threads (see arrows at 10:15:03 UT) were clearly seen to untwist during the eruption. Near the maximal phase of the flare, only a small segment of this piece of the filament was remained.

In Figure 3, the straight line at 00:08 UT indicates a piece of magnetic neutral line. The transverse field alignments show that the positive magnetic field (above the line) and the negative field were a single couple of magnetic features. It is identified from the history of flux evolution from MDI and HSOS magnetograms that they represented an emerging flux region (EFR) in this superactive region. The negative magnetic field squeezed upward to the left of the positive magnetic flux. During the 10 hour observations 


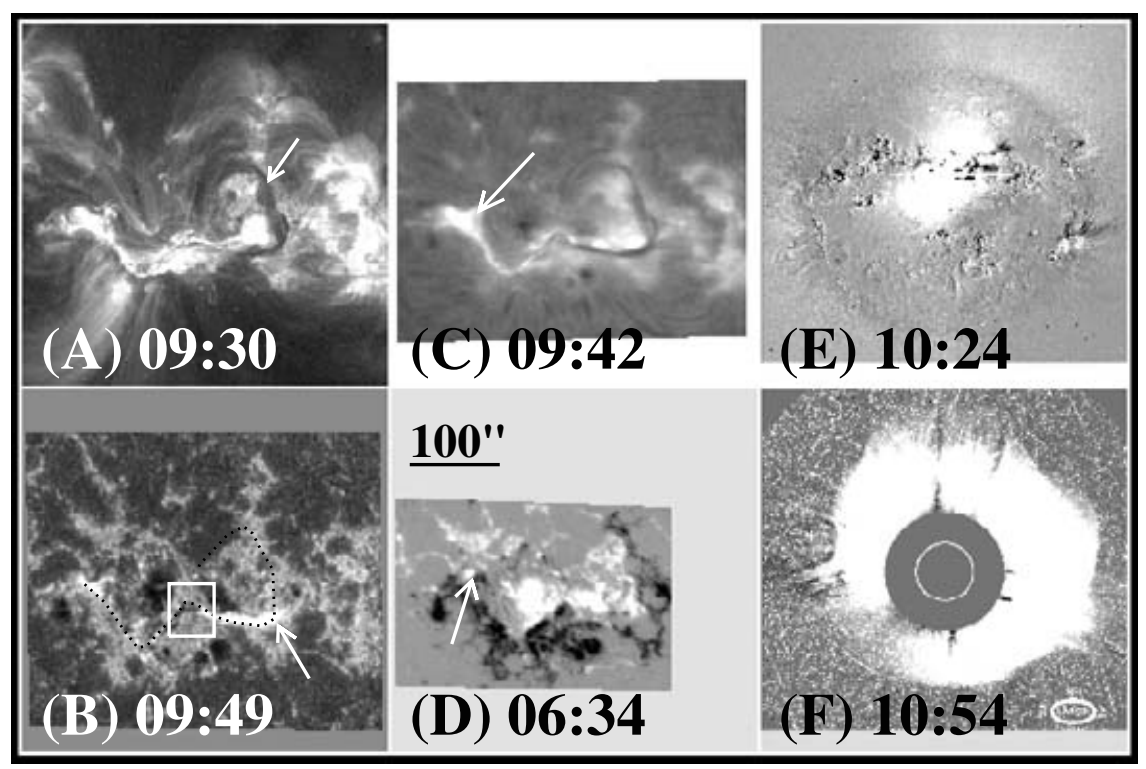

Figure 1. The appearance of the active phenomena in the region NOAA 9077 on July 14, 2000. A: a TRACE $195 \AA$ image; B: a TRACE $1600 \AA$ image; C: an H $\alpha$ filtergram; D: the corresponding line-of-sight magnetogram at 06:34 UT; E: a running difference of SOHO/EIT image. There is a bright patch of the X5.7 class flare; F: a running difference of LASCO C2 image shown a halo coronal mass ejection (Zhang et al. 2001).

from 23:32 UT July 13, the negative polarity field moved about $7.1 \times 10^{3} \mathrm{~km}$ (related to the negative polarity field shown by arrow ' 1 ' in Figure 3 ). The mean speed was $0.2 \mathrm{~km}$ $\mathrm{s}^{-1}$. The positive polarity field moved downward and canceled with a nearby negative field (indicated by a open square bracket at 01:01 UT) until the disappearance of negative flux. We have also noticed the flux cancellation when some positive magnetic patches (the two arrows at 01:01 and 04:14 UT) slid and intruded into the negative magnetic fields to its south. As a result of the shearing motion of opposite polarities in the EFR and related flux cancellation, the orientation of the magnetic neutral line altered obviously. During the period from 00:08 UT to 08:12 UT, its alignment changed $70^{\circ}$ (see the solid and dot lines at 08:12 UT, they represent the neutral lines at 08:12 and 00:08 UT, respectively).

Flux cancellation seen in vector magnetograms hints the magnetic reconnection in the photospheric level. Opposite polarity fields come from independent flux systems Transverse fields change alignment. Zhang et al.(2001) presented the first evidence that the slow magnetic reconnection in the lower atmosphere (in AR9077), which is manifested as observed flux cancellation, is of overwhelming importance in leading to the global instability responsible for the major magnetic activity.

\section{New flux emergence and CME origination}

Flux cancellation is always accompanied by flux emergence. Emerging flux regions (EFRs) are elementary building bricks of magnetic fields of ARs, and play a central role in explosive activity. Wang (2002) study the magnetic field evolution of AR 8100, the active region associated with four flare-CME events by vector magnetograms taken at Huairou. Some representative magnetograms are shown in Figure 4. At least five EFRs appeared since November 1 , and they added large amount of the magnetic flux to the main bipole. EFRs 1 and 2 emerged in the northern periphery of the region. EFR 2 


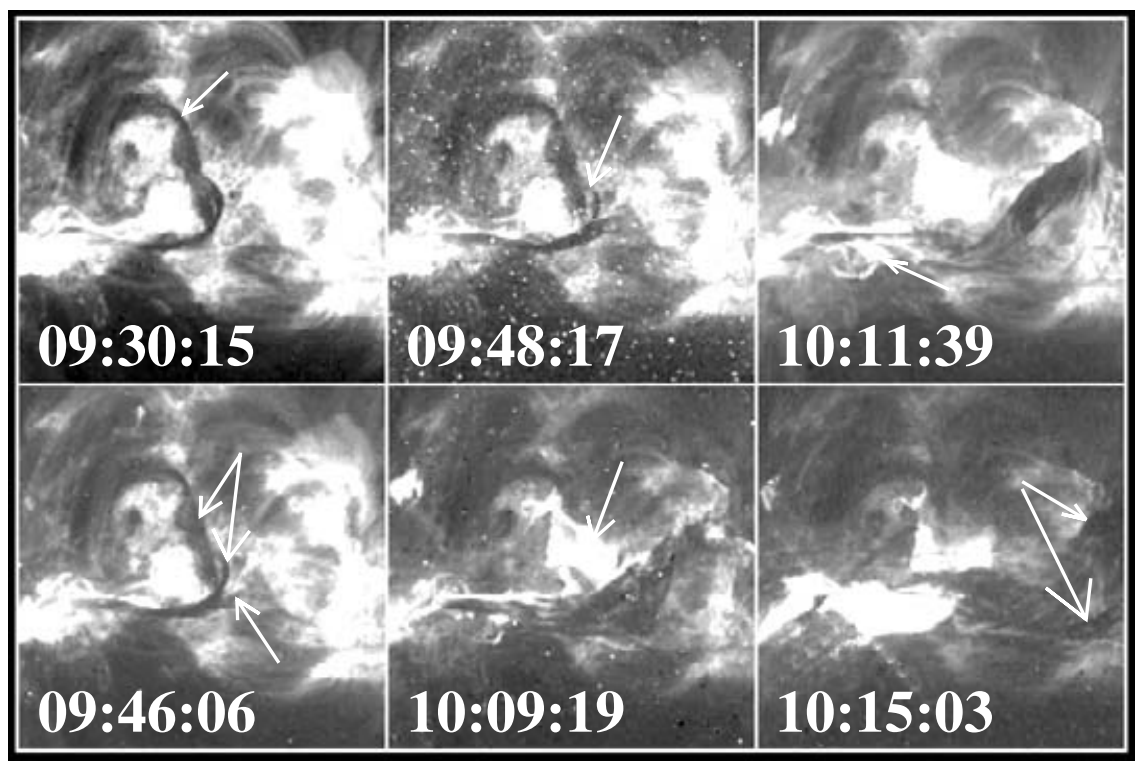

Figure 2. Time sequence of TRACE $195 \AA$ images shown the evolution of the filament. The field of view is about 290 by 290 square arcsec. The arrows in this figure are described in the text. (Zhang et al. 2001)

showed an exceptionally long duration and large separation. Its continuous growth and cancellation with the pre-existing negative flux in the north periphery (indicated by two arrows) were a central evolutionary feature of AR 8100. The flux cancellation was signified by an obvious decrease of negative flux on the eastern side of the magnetic neutral line, and an increase of EFR's positive flux on the western side. Note that the interface of EFR 2 and its impacted negative flux was characterized by the discontinuity in transverse field alignment. This is often seen when two topologically unconnected flux systems interact with each other. Flare-CME associated active regions always show obvious new flux emergence. In the example active region, AR 8100, all the CME-associated flares occurred close to EFR 2 that had exceptionally long duration and large separation.

\section{Helicity and CME initiation}

Emerging flux regions usually carry magnetic helicity. CMEs are thought to originate from the over accumulation of magnetic helicity (Low, 1996). Recent studies (Chae et al. 2001; Nindos \& Zhang, 2002; Demoulin et al. 2002, Green et al. 2002; Moon et al. 2002) revealed the incompetence of AR fields in creating enough helicity for CMEs. CME is a large-scale, or global scale activity. Its magnetic helicity must have been maintained in large or global scale fields.

Wang, Zhou, \& Zhang (2004)have tried to examine if particular helicity patterns are retained by CME-associated active regions (ARs). 9 ARs are selected and present complicated helicity patterns. Both helicity signs are seen in each AR as previously reported by Pevtsov, Canfield \& Metcalf (1994). For the 5 ARs with definitively identified key EFRs, it is found that the EFRs were born with the helicity of the sign opposite to the dominant helicity sign of the ARs. For the 2 ARs with somewhat uncertain EFRs, the results are the same. 

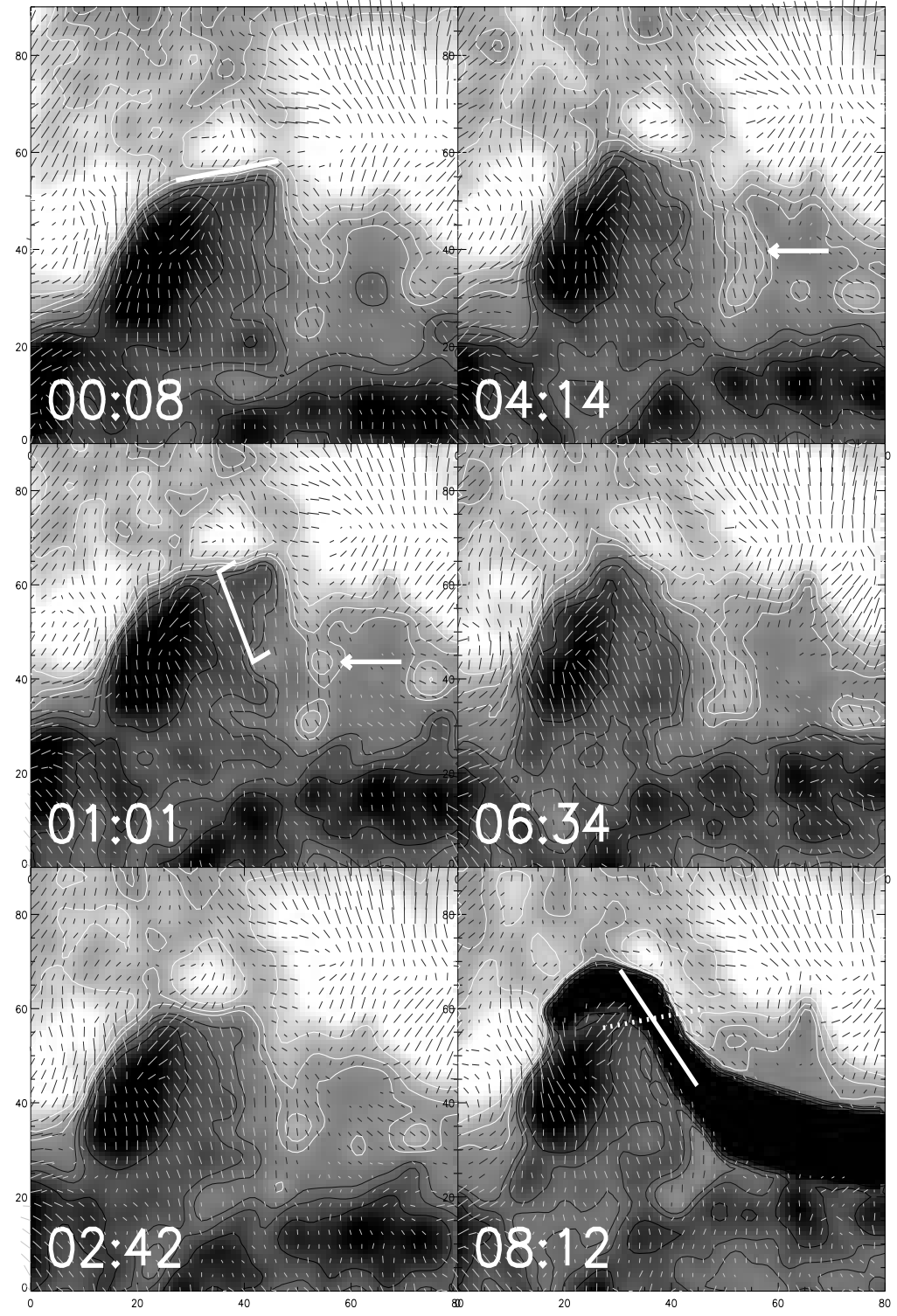

Figure 3. Time sequence of vector magnetograms showing the magnetic field evolution. On $x$ axis, 1 unit $=0.613$ arc sec, on y axis, 1 unit $=0.425$ arc sec (Zhang et al. 2001).

A famous CME-prolific AR in this solar cycle, AR8100, exhibited exactly particular helicity pattern. Liu et al.(2002) presented a helicity charging picture for this AR. They demonstrated that the magnetic reconnection between EFRs and pre-existing flux played a role in the formation of the sigmoidal structure of the AR. This appears to be contradictory to the findings in this work. Fortunately, very good time sequence of vector magnetograms were available to resolve this apparent contradiction. The EFR that was exemplified by Liu et al. (2002) was a smaller EFR emerged within the main positive sunspot and lasted only one day (see the lower-left panel of Fig.1 and the upper-right panels of Fig.2 of Liu et al. 2002). Wang, Zhou, \& Zhang (2004) show this EFR in the upper panel of our Figure 5 by a long arrow. It exhibited marginally the same sign of 


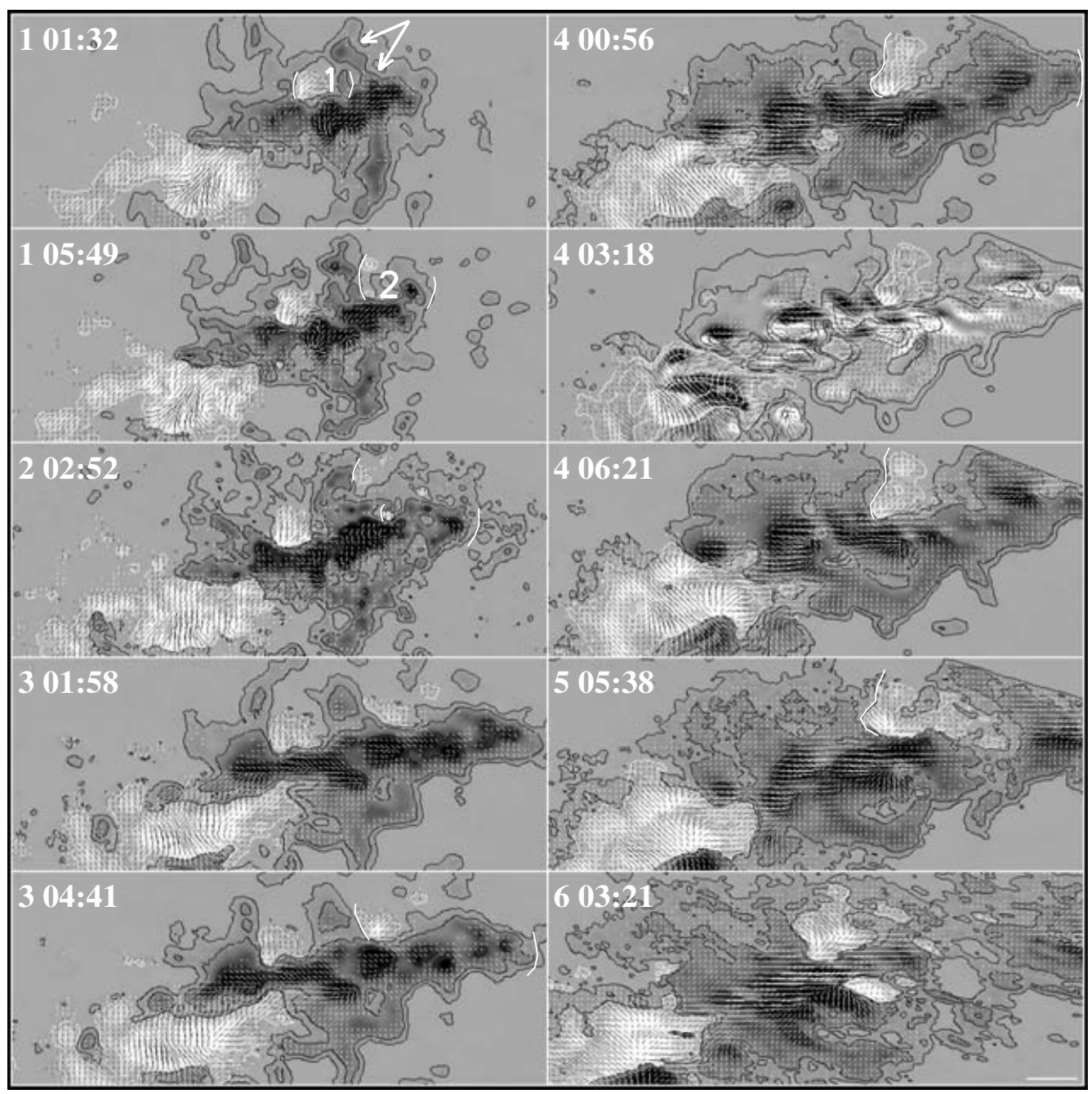

Figure 4. Vector magnetic field evolution of AR8100. Huairou vector magnetograms, which have been de-projected into the heliographic plane, are shown in time sequence. The longitudinal fields are given in gray-scale, i.e., lighter (darker) colors for positive (negative) polarity, and also in contours, whose levels are $\pm 50,250,1000,1500 \mathrm{G}$. The transverse fields are presented by short line segments with length proportional to field strength and alignment parallel to the field direction. Two EFRs are numbered as ' 1 ' and ' 2 ' in brackets which embrace opposite polarities of each EFR, respectively. For clear identification, EFR 2 was marked by brackets in several magnetograms. An image of current helicity $B_{z} J_{z}$ (at November 4, 03:18 UT) is also shown. The white bar at the right bottom corner indicates the scale of $30 \operatorname{arcsec}$ (Wang 2002).

helicity of the AR, and triggered a sizable, but confined flare. Moreover, it is also recognized that part of the magnetic flux in the main sunspot might come from EFRs which showed dominant negative helicity and played some roles in the formation of the complex topology of AR 8100. However, the key EFR (marked by the bracket in the magnetograms of Figure 5) which triggered explosive flares and homologous CMEs was an extraordinarily large and long-duration EFR. It grew for continuous 6 days. There were 6 homologous flare-CME events which appeared in the immediate interface between the positive flux of this EFR and pre-existing negative flux in the AR (Delannée \& Aulanier 1999). The 


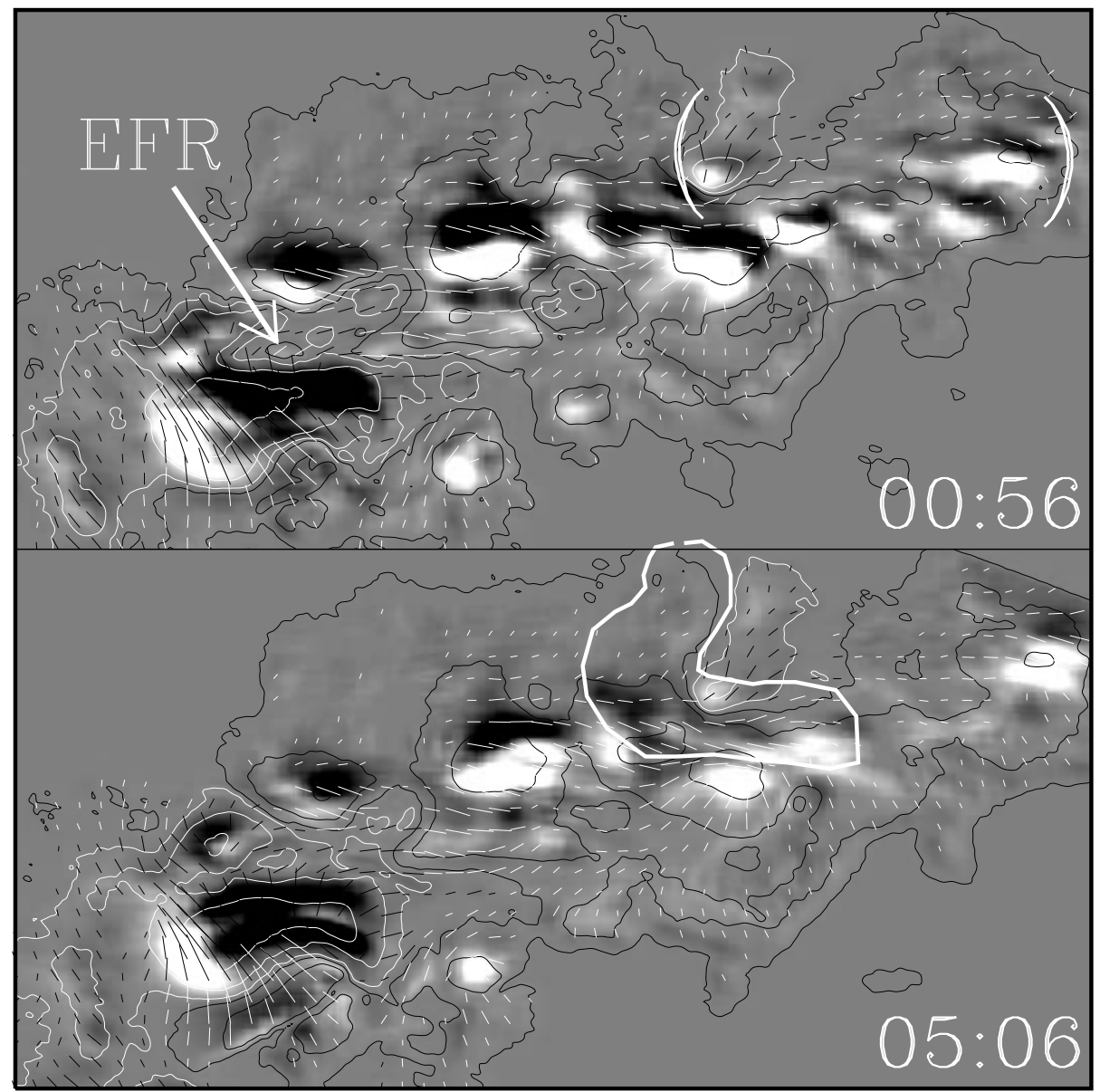

Figure 5. Helicity images of AR8100 scaled in the range of $\pm 20 A^{2} m^{-3}$, superposed by vector magnetograms with contours representing the flux density and short bars indicating the field azimuth. Light(dark) colors refer to positive(negative) helicity. The contour levels are $\pm 100,500,1500$ G. A long arrow indicates an EFR that was described by Liu et al.(2002). A bracket marks the key EFR; heavy white contours represents the flare ribbons at 05:52 UT of November 4 (Wang, Zhou, \& Zhang 2004).

flare ribbons of the major flare-CMEs that initiated from 05:52 UT is contoured in the lower panel of the figure. This key EFR exhibited predominant positive helicity during the whole flux emergence of 6 days. Thus, although it can not be excluded that some other EFRs may load the same sign helicity to the AR and caused confined flares, for the onset of flare-CME events the key EFR with opposite sign helicity seems to be necessary.

For 9 sampling ARs, contrary to the helicity charging picture, Wang, Zhou, \& Zhang (2004) find evidence that the new emerging flux often brings up the helicity with sign opposite to the dominant helicity of the ARs. This support the paradigm that interaction of topology-independent flux systems is a key ingredient in flare/CME magnetisms. Counter-helicity interaction causes the largest amount of magnetic free energy release, while co-helicity interaction results in the highest final energy state of the flux system. This idea is consistent with the 3D MHD simulation by Linton et al. (2001) and the helicity annihilation model by Kusano et al. (2003) 


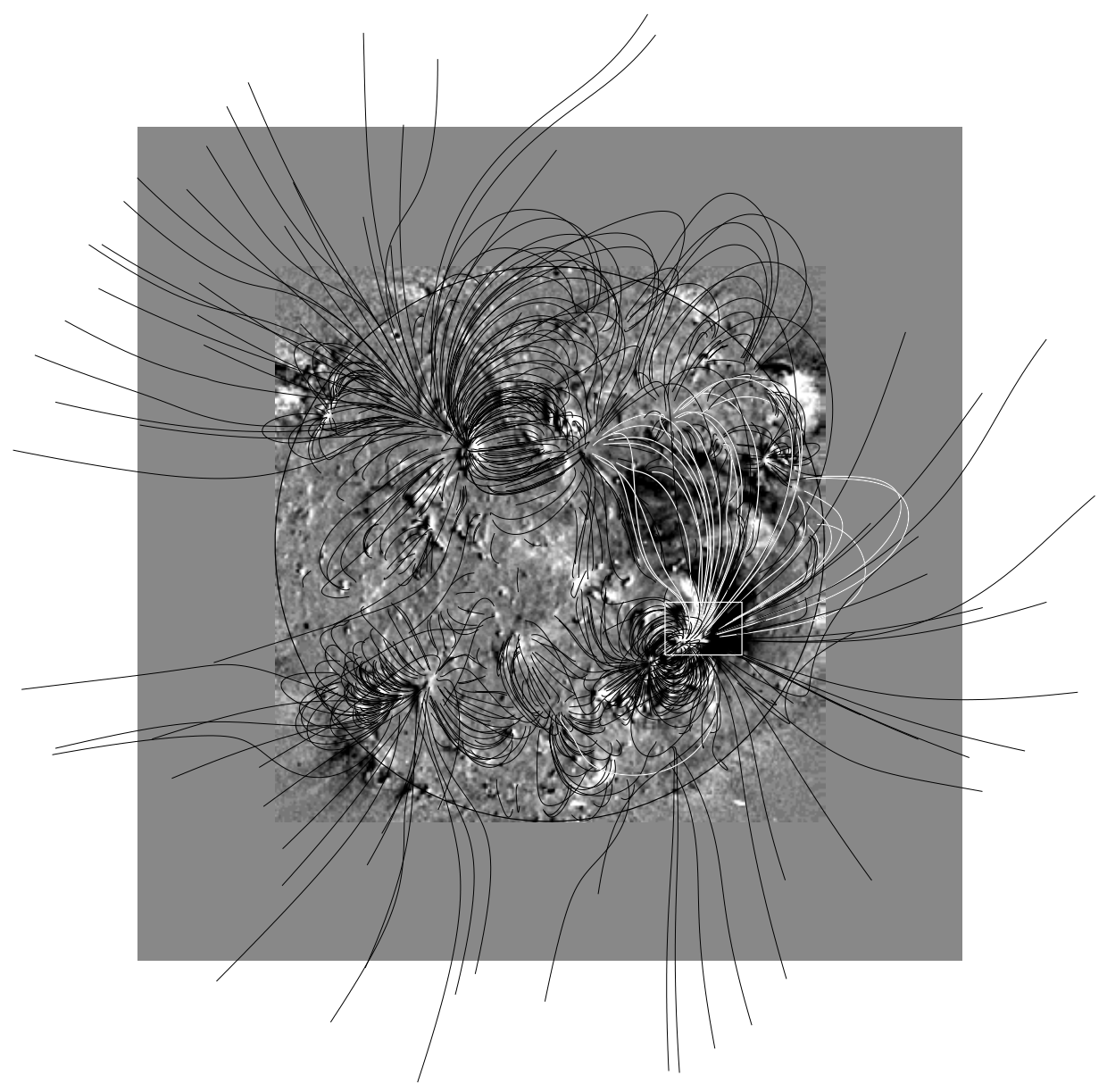

Figure 6. Parent magnetic structure of CMEs. Extrapolated 3-D magnetic lines of force superposed on an EIT difference image (06:15-05:41 UT) that shows EUV dimming of CME 3. This potential field extrapolation uses as the boundary condition an MDI magnetogram in the longitudinal range of -60 to 70 degrees and latitudinal range of -60 to 60 degrees. The vertical component is assumed to be equal to the longitudinal component in the data domain, outside which the flux density is set to zero (Wang 2002).

\section{Discussion}

In both the flare-associated and the flare/CME-associated active regions, evolving magnetic features are always the same. Why some flare-associated active regions have no CMEs? The magnetic field of AR 8100 as a whole showed a marked dominance of negative polarity. The total flux of the region in the observed field-of-view (see Figure 4) increased from $3 \times 10^{22}$ to $6 \times 10^{22}$ during November $1-6$, while the net flux varied in the range of $-(0.5-1.5) \times 10^{22} \mathrm{Mx}$. There must have been some large-scale magnetic loops connecting this region to other regions dominated by positive flux. This expectation was substantiated by potential field lines as extrapolated (using a boundary element method) on a MDI magnetogram of November 4 in Figure 6. They are plotted on an EIT difference image showing global dimming. The magnetic lines of force with height lower than 0.5 $\mathrm{R}_{\odot}$ and footpoint separation larger than $0.4 \mathrm{R}_{\odot}$ are highlighted in white. Spreading out from the vicinity of the flare site(see the box in the figure), they almost covered the whole dimming area. Wang (2002) identify these field lines with giant magnetic loops 
(GMLs). Similar large-scale interconnecting loops are reproduced by extrapolations on MDI data taken at other times during November 3-6. This leads us to suggest that the pre-CME magnetic skeleton be characterized by the GMLs that were vigorously impacted by smaller-scale magnetic loops (especially EFR 2) in AR 8100, in which the magnetic field was much stronger than that at the other end of the GMLs (see the volume density of magnetic lines of force). The global connectivity manifested by GMLs is also supported by the helicity distribution of AR 8100. The negative flux in the north periphery, canceling with EFR 2, had negative helicity, $B_{z} J_{z}$. Moreover, on average, AR 8100 had a negative current helicity (see the bottom part of Figure 5), which indicated a connection with the northern hemisphere (Canfield, Pevtsov, \& McClymont 1996). The structure of coronal magnetic fields over the active regions may be predominant! CMEassociated coronal magnetic fields exist: 1 . Large-scale magnetic connectivity; 2. Global magnetic non-potentiality and complexity, e.g., the total magnetic (current) helicity.

\section{Acknowledgements}

The work is supported by the National Natural Science Foundation of China (10233050) and National Key Basic Research Science Foundation (TG2000078404).

\section{References}

Burlaga, L. F., \& Behannon, K. W. 1982, Sol. Phys. 81, 181

Canfield, R.C., Pevtsov, A.A. \& McClymont, A.N. 1996, in Bentley, R.D. and Mariska, J.T. (eds.) Magnetic Reconnection in the Solar Atmosphere, PAS Conf. Series 111, p.341.

Chae, J., Wang, H., Qiu, J., Goode, P. R., Strous, L., \& Yun, H. S. 2001, ApJ 560, 476

Delannée, C., \& Aulanier, G. 1999, Sol. Phys. 190, 107

Démoulin, P., Mandrini, C. H., van Driel-Gesztelyi, L., Thompson, B. J., Plunkett, S., Kövari, Zs., Aulanier, G., \& Young, A. 2002b, A\&A 382, 650

Dere, K. P., Brueckner, G. E., Howard, R. A., Michels, D. J., \& Delaboudiniere, J. P. 1999, ApJ 516,465

Feynman, J., \& Martin, S. F. 1995, J. Geo. Res. 100, 3355

Green, L. M., Lopez Fuentes, M. C., Mandrini, C. H., Démoulin, P., van Driel-Gesztelyi, L., \& Culhane, J. L. 2002, Sol. Phys. 208, 43

Hudson, H. S., \& Cliver, E. W. 2001, J. Geo. Res. 106, 25199.

Kusano, K., Yokoyama, T., Maeshiro, T., \& Sakurai, T. 2003, Adv. Space Res. 32, 1931

Linton, M.G., Dahlburg, R. B., \& Antiochos, S. K. 2001, ApJ 553, 905

Livi S. H. B., Martin S. F., Wang H, \& Ai G. 1989, Sol. Phys. 121, 197

Liu Y., Zhao, X. P., Hoeksema, J. T., Scherrer, P. H., Wang, J., \& Yan, Y. 2002, Sol. Phys. 206, 333

Low, B. C. 1996, Sol. Phys. 167, 217

Martin S. F., Livi S. H. B., \& Wang J. 1985, Australian J. Phys. 38, 929

Moon, Y.-J., Chae, J., Wang, H., Choe, G. S., \& Park, Y. D. 2002, ApJ, 580, 528

Nindos, A., \& Zhang, H. 2002, ApJ 573, L133

Rust, D.W., \& Kumar, A. 1994, Sol. Phys. 155, 69

van Driel-Gesztelyi, L., Mandrini, C. H., Thompson, B., Plunkett, S., Aulanier, G., Démoulin, P., Schmieder, B., \& DeForest, C. 1999, ASP Conf. Ser. 184, 302

Wang, J., \& Shi, Z. 1993, Sol. Phys. 143, 119.

Wang, J. 2002, In Understanding Solar Active Phenomena (eds. J.-C. Henoux, C. Fang, \& N. Vilmer), Int. Sci. Publ. \& World Publ. Corp., Beijing, China, p.143

Wang, J., Zhou, G., \& Zhang, J. 2004, ApJ 615, 1021.

Zhang, J., Wang, J., Deng Y., \& Wu, D. 2001, ApJ 548, L99 


\section{Discussion}

Jun Lin: How much does the magnetic flux need to change in order to trigger a typical eruption?

ZHANG: About 5 10\% of the total flux of the active region.

SCHMIEDER: How do you relate the low atmosphere reconnection and the flare?

ZHANG: As at the site where flux cancellation takes place, brightening always appears, we consider the flux cancellation as low atmosphere reconnection. Now the direct connection between low atmosphere reconnection and a flare is still one of the unresolved problems.

Gopalswamy: You remarked that pre-eruption reconnection takes place at the photospheric level. What is the spatial and temporal relationship of pre-eruption reconnection and the reconnection in the corona associated with actual eruption?

ZHANG: The pre-eruption reconnection and the reconnection in the corona is always cospatial, but the reconnection in the corona is several hours later than the pre-eruption reconnection.

Yousef: When you have two magnetic loops of opposite field direction come in contact that will lead to magnetic annihilation and release of energy and would lead to disappearance of the photospheric magnetic field. So the magnetic annihilation may start high up in corona leading to field disappearance in photosphere. Can you comment on that?

ZHANG: Yes, sometime reconnection in corona leads to field disappearance in photosphere. However, flare onset and other solar activities always appear several hours later than flux cancellation observed in photosphere. This implies that flux cancellation may trigger coronal activities.

Koutchmy: Regarding the topology of the reconnection site, especially the case of the Bastille day 2000 flare, can you say something about the behavior of the horizontal component of the magnetic field near the site of apparent reconnection?

ZHANG: The horizontal component of this magnetic field is high shear, meaning almost parallel to the neutral line of the two reconnecting magnetic elements.

ShiBATA: What is the time scale of low atmospheric reconnection leading to global eruption? Have you observed velocity field associated with low atmospheric reconnection? If so, how large is the velocity?

ZHANG: Several tens of hours of low atmospheric reconnection lead to global eruption! We have not observed velocity field associated with low atmospheric reconnection! 